\title{
Experimental Evaluation of Multi-Agent Ontology Mapping Framework
}

\author{
Miklos Nagy and Maria Vargas-Vera
}

\begin{abstract}
Ontology mapping is a prerequisite for achieving heterogeneous data integration on the Semantic Web. The vision of the Semantic Web implies that a large number of ontologies are present on the Web that needs to be aligned before one can make use of them e.g. question answering on the Semantic Web. During the recent years a number of mapping algorithms, frameworks and tools have been proposed to address the problem of ontology mapping. Unfortunately comparing and evaluating these tools is not a straightforward task as these solutions are mainly designed for different domains. In this paper we introduce our ontology mapping framework called "DSSim" and present an experimental evaluation based on the tracks of the Ontology Alignment Evaluation Initiative (OAEI 2008).
\end{abstract}

\section{Multi Agent Ontology Mapping Framework}

As a requirement for the Semantic Web vision to become reality several difficulties have to resolved like ontology mapping, which makes it possible to interpret and align heterogeneous and distributed ontologies in this environment. For ontology mapping in the context of Question Answering over heterogeneous sources we propose a multi agent architecture [2] because as a particular domain becomes larger and more complex, open and distributed, a set of cooperating agents are necessary in order to address the ontology mapping task effectively. In real scenarios, ontology mapping can be carried out on domains with large number of classes and properties. Without the multi agent architecture the response time of the system can increase exponentially when the number of concepts to map increases.

\footnotetext{
Miklos Nagy

Knowledge Media Institute, The Open University, UK, e-mail: mn2336@student.open.ac.uk

Maria Vargas-Vera

Computing Department,The Open University, UK e-mail: m.vargas-vera@open.ac.uk
}

Please use the following format when citing this chapter:

Nagy, M. and Vargas-Vera, M., 2009, in IFIP International Federation for Information Processing, Volume 296; Artificial Intelligence Applications and Innovations III; Eds. Iliadis, L., Vlahavas, I., Bramer, M.; (Boston: Springer), pp. 145-150. 


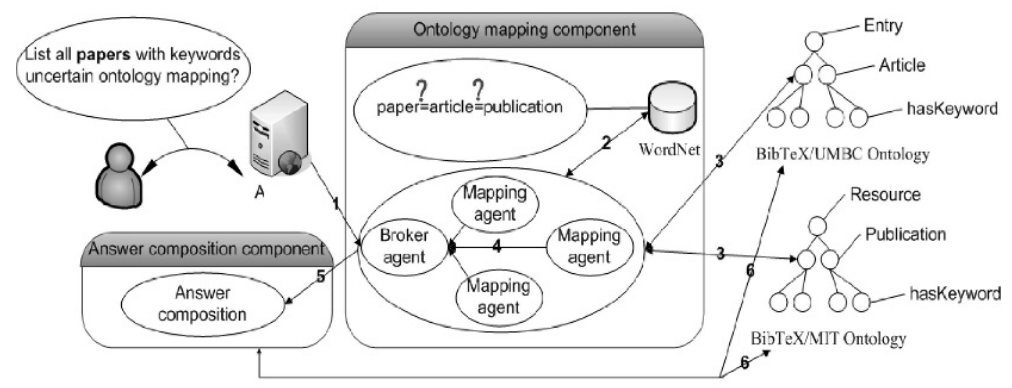

Fig. 1 Overview of the mapping system

An overview of our system is depicted on Fig. 1 The two real word ontologies ${ }^{12}$ describe BibTeX publications from the University of Maryland, Baltimore County (UMBC) and from the Massachusetts Institute of Technology (MIT) . The AQUA [7] system and the answer composition component are described just to provide the context of our work (our overall framework) but these are not our major target in this paper. The user poses a natural language query to the AQUA system, which converts it into FOL (First Order Logic) terms. The main components and its functions of the system are as follows. First broker agent receives FOL term, decomposes it(in case more than one concepts are in the query) and distributes the sub queries to the mapping agents. Mapping agents retrieve sub query class and property hypernyms from WordNet. and retrieve ontology fragments from the external ontologies, which are candidate mappings to the received sub-queries. Mapping agents use WordNet as background knowledge in order to enhance their beliefs on the possible meaning of the concepts or properties in the particular context. At this point mapping agents build up coherent beliefs by combining all possible beliefs over the similarities of the sub queries and ontology fragments. Mapping agents utilize both syntactic and semantic similarity algorithms build their beliefs over the correctness of the mapping. After this step broker agent passes the possible mappings into the answer composition component for particular sub-query ontology fragment mapping in which the belief function has the highest value. In the last step the answer composition component retrieves the concrete instances from the external ontologies or data sources, which will be included into the answer and creates an answer to the user's question.

The organisation of this paper is as follows. In section 2 we analyse related systems, which have participated in more than 3 OAEI tracks. In section 3 we present our experimental evaluation of the benchmarks, anatomy and library tracks. Finally in section 4 we draw our conclusions of our evaluation.

\footnotetext{
${ }^{1} \mathrm{http}: / /$ ebiquity.umbc.edu/ontology/publication.owl

${ }^{2}$ http://visus.mit.edu/bibtex/0.01/bibtex.owl
} 


\section{System Analysis and Related Work}

Several ontology mapping systems have been proposed to address the semantic data integration problem of different domains independently. In this paper we consider only those systems, which have participated in the OAEI competitions and has been participated more than two tracks. There are other proposed systems as well however as the experimental comparison cannot be achieved we do not include them in the scope of our analysis. Lily [8] is an ontology mapping system with different purpose ranging from generic ontology matching to mapping debugging. It uses different syntactic and semantic similarity measures and combines them with the experiential weights. Further it applies similarity propagation matcher with strong propagation condition and the matching algorithm utilises the results of literal matching to produce more alignments. In order to assess when to use similarity propagation Lily uses different strategies, which prevents the algorithm from producing more incorrect alignments. ASMOV [1] has been proposed as a general mapping tool in order to facilitate the integration of heterogeneous systems, using their data source ontologies. It uses different matchers and generates similarity matrices between concepts, properties, and individuals, including mappings from object properties to datatype properties. It does not combine the similarities but uses the best values to create a pre alignment, which are then being semantically validated. Mappings, which pass the semantic validation will be added to the final alignment. ASMOV can use different background knowledge e.g. Wordnet or UMLS Metathesaurus(medical background knowledge) for the assessment of the similarity measures. RiMOM [6] is an automatic ontology mapping system, which models the ontology mapping problem as making decisions over entities with minimal risk. It uses the Bayesian theory to model decision making under uncertainty where observations are all entities in the two ontologies. Further it implements different matching strategies where each defined strategy is based on one kind of ontological information. RiMOM includes different methods for choosing appropriate strategies (or strategy combination) according to the available information in the ontologies. The strategy combination is conducted by a linear-interpolation method. In addition to the different strategies RiMOM uses similarity propagation process to refine the existing alignments and to find new alignments that cannot be found using other strategies. RiMOM is the only system other than DSSim in the OAEI contest that considers the uncertain nature of the mapping process however it models uncertainty differently from DSSim. RiMOM appeared for first time in the OAEI-2007 whilst DSSim appeared in the OAEI-2006.

\section{Experimental Analysis}

Experimental comparison of ontology mapping systems is not a straightforward task as each system is usually designed to address a particular need from a specific domain. This problem has been acknowledged by the Ontology Mapping community 
and as a response to this need the Ontology Alignment Evaluation Initiative ${ }^{3}$ has been set up in 2004. The evaluation was measured with recall, precision and Fmeasure, which are useful measures that have a fixed range and meaningful from the mapping point of view. The experiments were carried out to assess the efficiency of the mapping algorithms themselves. The experiments of the question answering (AQUA) using our mappings algorithms are out of the scope of this paper. Our main objective was to compare our system and algorithms to existing approaches on the same basis and to allow drawing constructive conclusions.

\subsection{Benchmarks}

The OAEI benchmark contains tests, which were systematically generated starting from some reference ontology and discarding a number of information in order to evaluate how the algorithm behave when this information is lacking. The bibliographic reference ontology (different classifications of publications) contained 33 named classes, 24 object properties, 40 data properties. Further each generated ontology was aligned with the reference ontology. The benchmark tests were created and grouped by the following criteria. Group 1xx are simple tests such as comparing the reference ontology with itself, with another irrelevant ontology or the same ontology in its restriction to OWL-Lite. Group 2xx are systematic tests that were obtained by discarding some features from some reference ontology e.g. name of entities replaced by random strings, synonyms, name with different conventions. Group 3xx contain four real-life ontologies of bibliographic references that were found on the web e.g. BibTeX/MIT, BibTeX/UMBC. Figure 2 shows the 6 best performing systems out of 13 participants. We have ordered the systems based on the their the F-Value of the H-means because the H-mean unifies all results for the test and F-Value represents both precision and recall.

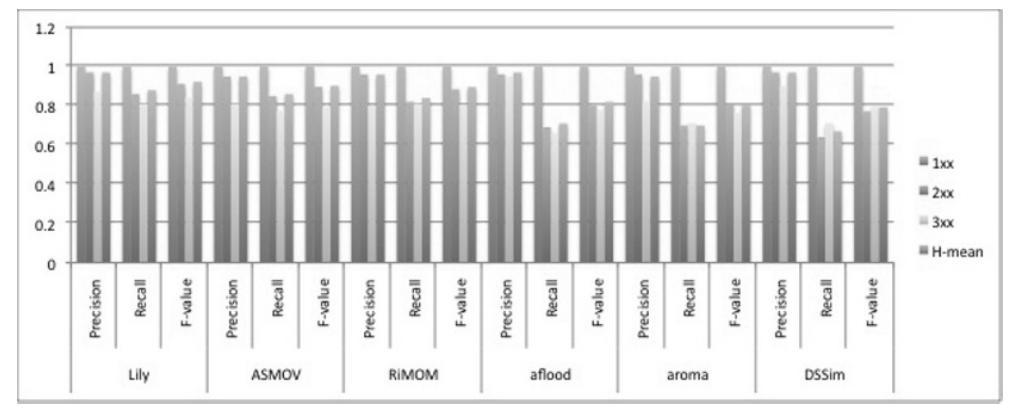

Fig. 2 Best performing systems in the benchmarks track based on H-mean and F-value

\footnotetext{
${ }^{3}$ http://oaei.ontologymatching.org/
} 
In the benchmark test we have performed in the upper mid range compared to other systems. Depending on the group of tests our system compares differently to other solutions. For the Group 1xx our results are nearly identical to the other systems. In the group $2 x x$ tests where syntactic similarity can determine the mapping outcome our system is comparable to other systems. However where semantic similarity is the only way to provide mappings our systems provides less mappings compared to the other systems in the best six. For the tests in group 3xx considering the F-value only 3 systems SAMBO, RIMOM and Lily performed better than DSSim. The weakness of our system to provide good mappings when only semantic similarity can be exploited is the direct consequence of our mapping architecture. At the moment we are using four mapping agents where 3 carries our syntactic similarity comparisons and only 1 is specialised in semantics. However it is worth to note that our approach seems to be stable compared to our last year's performance as our precision recall values were similar in spite of the fact that more and more difficult tests have been introduced in this year. As our architecture is easily expandable with adding more mapping agents it is possible to enhance our semantic mapping performance in the future.

\subsection{Library}

The objective of this track was to align two Dutch thesauri used to index books from two collections held by the National Library of the Netherlands. Each collection is described according to its own indexing system and conceptual vocabulary. On the one hand, the Scientific Collection is described using the GTT, a huge vocabulary containing 35,000 general concepts ranging from Wolkenkrabbers (Sky-scrapers) to Verzorging (Care). On the other hand, the books contained in the Deposit Collection are mainly indexed against the Brinkman thesaurus, containing a large set of headings (more than 5,000) that are expected to serve as global subjects of books. Both thesauri have similar coverage (there are more than 2,000 concepts having exactly the same label) but differ in granularity. For each concept, the thesauri provide the usual lexical and semantic information: preferred labels, synonyms and notes, broader and related concepts, etc. The language of both thesauri is Dutch, but a quite substantial part of Brinkman concepts (around 60\%) come with English labels. For the purpose of the alignment, the two thesauri have been represented according to the SKOS model, which provides with all these features.

In the library track DSSim has performed the best out of the 3 participating systems. The track is difficult partly because of its relative large size and because of its multilingual representation. However these ontologies contain related and broader terms therefore the mapping can be carried out without consulting multi lingual background knowledge. This year the organisers have provided instances as separate ontology as well however we did not make use of it for creating our final mappings. For further improvements in recall and precision we will need to consider these additional instances in the future. 


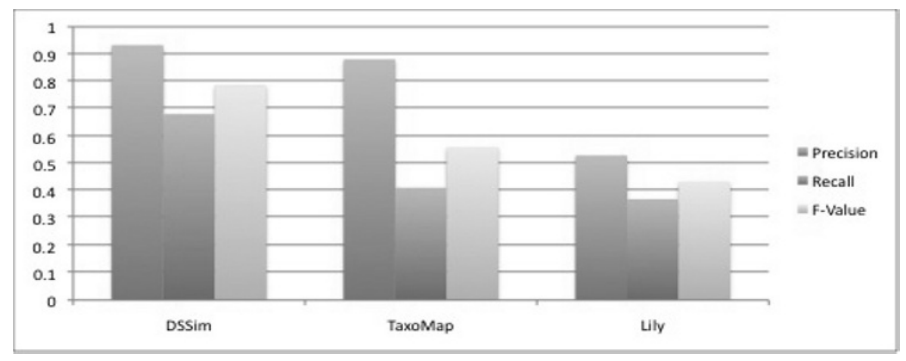

Fig. 3 All participating systems in the library track ordered by F-value

\section{Conclusions}

In this paper we have analysed two different experimental tests that were carried out in order to evaluate our integrated ontology mapping solution. We have showed that our solution DSSim, which is the core ontology mapping component of our proposed multi agent ontology mapping framework performs really well compared to other solutions. The analysis of other OAEI 2008 tracks in which we have participated are out of the scope of this paper, however, a detailed description of the other tracks can be found in [5]. Nevertheless we continuously evaluate the performance of our system through OAEI competitions $[3,4,5]$ that allows us to improve, evaluate and validate our solution compared to other state of the art systems. So far our qualitative results are encouraging therefore we aim to investigate further the belief combination optimisation, compound noun processing and agent communication strategies for uncertain reasoning in the future.

\section{References}

1. Yves R. Jean-Mary and Mansur R. Kabuka. Asmov: Ontology alignment with semantic validation. In Joint SWDB-ODBIS Workshop, 2007.

2. Miklos Nagy, Maria Vargas-Vera, and Enrico Motta. Multi agent ontology mapping framework in the aqua question answering system. In MICAI 2005: Advances in Artificial Intelligence, 4th Mexican International Conference on Artificial Intelligence, pages 70-79, 2005.

3. Miklos Nagy, Maria Vargas-Vera, and Enrico Motta. Dssim-ontology mapping with uncertainty. In The 1st International Workshop on Ontology Matching, 2006.

4. Miklos Nagy, Maria Vargas-vera, and Enrico Motta. Dssim - managing uncertainty on the semantic web. In The 2nd International Workshop on Ontology Matching, 2007.

5. Miklos Nagy, Maria Vargas-Vera, and Piotr Stolarski. Dssim results for oaei 2008. In The 3rd International Workshop on Ontology Matching, 2008.

6. Jie Tang, Juanzi Li, Bangyong Liang, Xiaotong Huang, Yi Li, and Kehong Wang. Using bayesian decision for ontology mapping. Web Semantics, 2006.

7. Maria Vargas-Vera and Enrico Motta. Aqua - ontology-based question answering system. In Third International Mexican Conference on Artificial Intelligence (MICAI-2004), 2004.

8. Peng Wang and Baowen Xu. Lily: Ontology alignment results for oaei 2008. In The 3rd International Workshop on Ontology Matching, 2008. 